\title{
To Study the Correlation of Embryo and Oocyte Quality with Clinical Pregnancy Rate
}

\section{Revati Sunil Thorat ${ }^{1}$, Aakash More ${ }^{2}$, Mehul Salve ${ }^{3}$, Deepti Shrivastava ${ }^{4}$}

'M.Sc. Clinical Embryology, Department of Anatomy, Jawaharlal Nehru Medical College, Datta Meghe lnstitute of Medical Sciences, Wardha, Maharashtra, India; 'Senior Embryologist Wardha Test Tube Baby Centre A.V.B.R.H,Jawaharlal Nehru Medical College, Datta Meghe Institute of Medical Sciences, Wardha, Maharashtra, India; ${ }^{3}$ Associate Professor, Department of OBGY, Jawaharlal Nehru Medical College, Datta Meghe Institute of Medical Sciences, Wardha, Maharashtra, India; 4 Professor, Department of OBGY, Jawaharlal Nehru Medical College, Datta Meghe Institute of Medical Sciences, Wardha, Maharashtra, India.

\section{ABSTRACT}

Background: Embryo and oocyte grading or scoring is the selection and classification of embryo and oocyte according to its quality. Embryo quality is an important factor for successful delivery. It is a non-invasive method of embryo evaluation. It is an easy assessment of embryo without any harm which includes morphological observation. A well morphologically graded oocyte improves the quality of the embryo.

Objectives: This study aims to use a standard grading system for better morphological assessment of oocytes and embryos. Eventually, the correlation of oocyte and embryo quality with pregnancy outcome will be determined.

Methodology: This non-invasive methodology includes the recording of treatment history and the indications. Counselling of the participants for research will be done. The further protocol includes the morphological assessment of oocyte as well as an embryo by grading system (Gardner) and $\beta$ hCG level will be evaluated.

Expected Results: 50 women will be included. Embryo and oocyte grading system by Gardner will be used for morphological analysis of embryos at cleavage as well as at blastocyst stage according to the day of transfer. Gardner oocyte grading/scoring system may predict the selection of best oocyte for IVF/ICSI. Further Gardner embryo grading system may predict the best morphological evaluation of embryo and eventually selection of best quality embryo for transfer. Thus by using the above grading method for selection of embryo for transfer, implantation and clinical pregnancy rate may increase.

Key Words: Oocyte, Embryo grading system, Non-invasive methodology, Embryo quality, Morphology, Pregnancy Rate

\section{INTRODUCTION}

Infertility is the inability to conceive a child. It has different causes including male as well as female factors. It is one of the major health care problems in society. To overcome this problem infertility treatments are carried out which includes techniques like IVF, ICSI etc. The selection of embryo for embryo transfer is a significant step. It has always been challenging for embryologists to select a proper embryo. The quality of the embryo is one of the predictors of successful implantation of the embryo. The transfer of poor quality of embryo may lead to abortion and decrease in clinical pregnancy rate.

The embryo grading system is a method of selection and classification of the embryo as well as oocyte according to its quality. Embryo grading is one of the predictive parameters for successful pregnancy outcome. This preimplantation embryo observation is very important. For achieving successful delivery, selection of best quality embryo is necessary for ART. Selection of best embryo reduces the number of embryos which transferred and also reduces the number of multifetal pregnancies. ${ }^{2}$ Embryo grading is a method which includes and emphases observation of embryos. It is an evaluation of embryos which is non-invasive and can be carried out without harming the embryos. ${ }^{2}$ The selection of better embryo leads to better endometrial receptivity. It reduces the requirement to transfer multiple embryos to achieve successful delivery. Clinically it is crucial to lessen the variability in embryo scoring because this grading of embryo predicts the successful treatment and decides which embryo to be transferred. ${ }^{3}$ The embryo grading system has benefits like, 1 .

\section{Corresponding Author:}

Aakash More, Senior Embryologist, Wardha Test Tube Baby Centre A.V.B.R.H, Jawaharlal Nehru Medical College, Datta Meghe Institute of Medical Sciences, Wardha, Maharashtra, India; Mob: 8668969691; Email: aakashmore87@gmail.com

ISSN: 2231-2196 (Print)

Received: 22.09 .2020
ISSN: 0975-5241 (Online)

Revised: 25.10 .2020
Accepted: 08.11.2020
Published: 30.11 .2020 
Accurate selection of embryo before implantation. 2. Reduction of chances of multiple pregnancies and 3. Evaluation of proper culture media for embryo. ${ }^{4}$ Grading of embryos leads to good synchronization of embryo and endometrium and thus selection of better quality graded embryo may lead to high implantation rates. The stage and grade of the embryo may have an essential role in successful pregnancy. ${ }^{4}$

Embryo examination is an observation which is based on morphology and variations of embryo development. Selection of better quality embryo includes the following parameters,

- Pronuclear morphology

- Appearance of cytoplasm

- Early cleavage

- Size, symmetry and fragmentation of blastomeres

- Multinucleation in each blastomere

- Compaction and expansion of blastomere ${ }^{1}$

Additionally, oocyte grading is also as important as embryo grading is. Oocyte increases the quality of the embryo. Highest oocyte scoring might be useful for prediction of high clinical pregnancy rate. For better morphology of embryo, oocyte should be of better quality and normal morphology. Morphological evaluation of oocyte is less appreciated. Thus focus on oocyte scoring may help to increase embryo quality and eventually it may help in better implantation of embryo. ${ }^{6}$ Following parameters of oocytes are observed while grading of oocyte,

- The amorphic shape of oocyte

- Granularity in the perivitelline space

- Inclusions

- Vacuolization

- The dark colour of the cytoplasm

- Changes in the colour and construction of the zona pellucida

- Changes in the shape and size of the polar body (Gardener)

The purpose of this study is to evaluate the relationship between embryo morphology and the success rate of implantation of the embryo. Embryo quality may be the major reason for implantation failure thus embryo grading may help to increase the success rate of embryo implantation.

In a few studies, Embryo scoring systems are explained which are widely used. It has been described how a noninvasive embryo evaluation methods are useful in ART. The relation of advanced embryo classification with ART success rates is described. This study focuses on the preimplantation morphological evaluation of embryo. ${ }^{2}$ Complimentarily, In a few studies, A simplified blastocyst grading system and its clinical importance have been described. This study focuses on prognostic potential, inter-observer and intraobserver variability of described blastocyst grading system. This study shows that the grading system determines the clinical out- come of implantation by classifying high-quality blastocyst which ultimately gives the positive result of implantation than poor-quality blastocycst. ${ }^{3}$

Certain studies show that scoring of the embryo is important at different phases before implantation. It has mentioned in the few studies that it is essential to analyze the embryo at both zygotes as well as at the pre-implantation stage. Factors like a cytoplasmic halo, alignment of nuclear precursor body at the zygote stage and blastomere size and its equality at cleavage stage are important for evaluation. For blastocysts expansion and cell number are important factors. ${ }^{4}$ Simultaneously few studies focus on how oocyte scoring helps to achieve a good embryo scoring. This study shows the relation between oocyte quality and developing embryo quality. In a few studies, it is showed that the grade of the embryo can determine the live birth rate as well as implantation. The grading of embryos can be used to predict the number of embryos for transfer. It has stated that cleavage-stage cannot determine the clinical pregnancy outcome. ${ }^{5}$ It has been predicted in this study that oocyte scoring might help to improve embryo quality which may increase the clinical pregnancy rate. ${ }^{6}$

\section{OBJECTIVES}

We hypothesized that embryo quality may be one of the reasons for the failure of embryo implantation. Thus high-quality embryo may implant and may give high implantation. ${ }^{5}$ To achieve this we set the following objectives,

- To assess the role stimulation protocol on oocyte quality.

- Observation and grading of oocytes after oocyte retrieval according to its morphology.

- Observation and grading of embryos on day 3 or day 5 .

- Selection of better quality of embryo to transfer according to its grading.

- Prediction of the outcome by checking $\beta$ hCG level.

\section{MATERIALS AND METHODS}

This Observational Study will be done in Wardha test tube baby centre AVBRH (SAWANGI) WARDHA. Relevant data on the demographics and treatment history as well as the indications will be recorded. Counselling of all participants for research work will be done. Written as well as verbal consent will be taken from the participants. The routine protocol in our set up is as follows: History of the patient will be taken.

Screening of the patients will be done which are suitable for research work by considering the parameters such as previous treatment, indications, BMI, age etc. A proper verbal, as well as written consent for research work, will be taken from the patients that are enrolled for research work. 
Before the ovum aspiration procedure, according to the standard stimulation protocol, ovarian stimulation will be given to the patient from day 2 of menstruation. Ovum aspiration procedure will be performed on day 10/12 of menstruation and oocyte screening will be performed. Oocyte morphological analysis (Gardner) will be performed before IVF/ ICSI procedure by considering characteristics such as oocyte size, shape, polar body, perivitelline space, zona pellucida, granulation, cytoplasmic parameters like vacuoles, refractile bodies, smooth endoplasmic reticulum etc. The oocyte with the best morphology will be selected for ICSI/IVF.

After considering male as well as female factors, recommended procedure ICSI/IVF will be performed. The graded or scored oocyte will be used for the recommended procedure.

According to the described embryo grading system, the morphological evaluation of embryo will be performed. The characteristics like trophectoderm, ICM- Inner Cell Mass, fragmentation etc will be considered and analysed. Eventually, the embryo with the best morphology will be selected for embryo transfer.) (After morphological observation of embryo, the morphologically best embryo will be selected and will be transferred. Analysis of pregnancy outcome and comparing it with a grade of the transferred embryo concerning stimulation protocol.

Study Setting: Study will be conducted at Wardha Test Tube Baby Centre, AVBRH planned to recruit patients between from August 2019 to August 2021

\section{Participants:}

\section{Inclusion Criterial:}

- Infertile couple attending WARDHA TEST TUBE BABY CENTRE, AVBRH

\section{Exclusion Criterial:}

- Patients not giving consent for research.

- Patients having infections like HIV, HbsAG etc.

- Excluding patients with male factor responsible for infertility.

Study size: 50 infertile couple based on the following formula,

$$
\mathrm{N}=\underline{\underline{\underline{2}}} \frac{* \mathrm{~N} * \mathrm{p}(1-\mathrm{p})}{\mathrm{C}^{2}(\mathrm{~N}-1)+\mathfrak{x}^{2} \mathrm{p}(1-\mathrm{p})}
$$

Total population, $\mathrm{N}=120$ during 36 months

$\aleph^{2}=$ Chi-square value for 1 degrees at some desired probability level. This is 3.84 at $5 \%$ level of significance

$\mathrm{P}=50 \%$ proportion

$\mathrm{Q}=100-\mathrm{p}$

$=50$
$\mathrm{C}=$ Confidence interval of the one choice $(95 \% \mathrm{CI})$

$=0.05$

$\mathrm{N}=\underline{3.84 * 120 * 0.5 * 0.5}$

$(0.05) 2 * 24+3.84 *(0.5 * 0.5)$

$=50$

\section{EXPECTED OUTCOMES}

Total 50 women will be included. Embryo and oocyte grading system by Gardner will be used for morphological analysis of embryos at cleavage as well as at blastocyst stage according to the day of transfer. Gardner oocyte grading/scoring system may predict the selection of best oocyte for IVF/ ICSI. Further Gardner embryo grading system may predict the best morphological evaluation of embryo and eventually selection of best quality embryo for transfer. Thus by using the above grading method for selection of embryo for transfer, implantation and clinical pregnancy rate may increase.

\section{DISCUSSION}

Embryo grading system aims to select an ideal embryo for transfer. According to previous studies, embryo quality increases the implantation rate and so clinical pregnancy. Few studies also state that oocyte scoring improves embryo quality and embryo scoring. Selection of good quality oocyte may lead to a better quality embryo. By using Gardner oocyte and embryo grading system good quality embryo may be selected for transfer and there may be an increase in implantation and clinical pregnancy rate. It may help to reduce multifetal pregnancies by selecting the best quality embryo which may emphasize single embryo transfer. Several related studies were reviewed. Reflections from the global burden of disease study are evident. ${ }^{7-9}$. Sawal et. al. studied the effect of feminine obesity on the outcome of the oocyte in subfertile females. ${ }^{10}$ Singh et al. conducted a Comparative study of transferring a frozen embryo with short culture versus overnight culture and also assess the prevalence rate of psychological distress among infertility patient. ${ }^{11}$

\section{ACKNOWLEDGMENT}

Authors acknowledge the immense help received from the scholars whose articles are cited and included in references to this manuscript. The authors are also grateful to authors / editors / publishers of all those articles, journals, and books from which the literature for this article has been reviewed and discussed.

\section{Conflict of Interest: Nil}




\section{Source of Funding: Nil}

\section{REFERENCES}

1. Wintner EM, Hershko-Klement A, Tzadikevitch K, Ghetler Y, Gonen O, Wintner O, et al. Does the transfer of a poor quality embryo together with a good quality embryo affect the In Vitro Fertilization (IVF) outcome? J Ovarian Res 2017 Dec;10(1):1-5.

2. Bączkowski T, Kurzawa R, Głąbowski W. Methods of embryo scoring in vitro fertilization. Reproduct Biol 2004;4(1):5-22.

3. Richardson A, Brearley S, Ahitan S, Chamberlain S, Davey T, Zujovic L, et al. A clinically useful simplified blastocyst grading system. Reproduct Biomed 2015 Oct ;31(4):523-30.

4. Nasiri N, Eftekhari-Yazdi P. An overview of the available methods for morphological scoring of pre-implantation embryos in vitro fertilization. Cell J 2015;16(4):392.

5. Dennis SJ, Thomas MA, Williams DB, Robins JC. Embryo morphology score on day 3 is predictive of implantation and live birth rates. J Assis Reproduct Genet 2006;23(4):171-5.

6. Lazzaroni-Tealdi E, Barad DH, Albertini DF, Yu Y, Kushnir VA, Russell $\mathrm{H}$, et al. Oocyte scoring enhances embryo-scoring in predicting pregnancy chances with IVF where it counts most. PLoS One 2015;10(12):e0143632.

7. James SL, Castle CD, Dingels ZV, Fox JT, Hamilton EB, Liu Z, et al. Estimating global injuries morbidity and mortality: methods and data used in the Global Burden of Disease 2017 study. Inj Prev 2020;26(1):i125-53.

8. Murray CJL, Abbafati C, Abbas KM, Abbasi M, Abbasi-Kangevari M, Abd-Allah F, et al. Five insights from the Global Burden of Disease Study 2019. Lancet 2020;396(10258):1135-59.

9. Wang H, Abbas KM, Abbasifard M, Abbasi-Kangevari M, Abbastabar H, Abd-Allah F, et al. Global age-sex-specific fertility, mortality, healthy life expectancy (HALE), and population estimates in 204 countries and territories, 1950-2019: a comprehensive demographic analysis for the Global Burden of Disease Study 2019. Lancet 2020;396(10258):1160-203.

10. Sawal A, Gajbe U, Singh B, More A, Bankar N. Effect of Feminine Obesity on the Outcome of Oocyte in Subfertile Females. Int J Cur Res Rev 2020;12(14).

11. Singh BR, More A, Ambad RS, Bankar N, Ghogare AS. Comparative study of transferring frozen embryo with short culture versus overnight culture and also assess prevalence rate of psychological distress among infertility patient. Int J Res Pharma Sci 2020;11(2):2489-92. 